\section{SOI: 1.1/TAS DOI: 10.15863/TAS International Scientific Journal Theoretical \& Applied Science}

\author{
p-ISSN: 2308-4944 (print) e-ISSN: 2409-0085 (online) \\ Year: $2015 \quad$ Issue: $05 \quad$ Volume: 25
}

Published: $30.05 .2015 \quad$ http://T-Science.org
Kanat Mukashevich Mukashev prof., DrSc,

Al-Farabi Kazakh National University, Kazakhstan mukash_kanat@mail.ru

Gulmira Shariphovna Yar-Mukhamedova prof., DrSc,

Al-Farabi Kazakh National University, Kazakhstan gulmira-alma-ata@mail.ru

Kunsulu Seidazovna Shadinova as. prof., $\mathrm{PhD}$,

Asfendiarov Kazakh National Medical University, Kazakhstan

Gulnara Toktanaevna Shoinbaeva Senior Lecturer, Abai Kazakh National University, Kazakhstan

\title{
ANNIHILATION OF POSITRONS IN RADIATION DAMAGED TITAN ALLOYS
}

Abstract: Application of positron spectroscopy method for research of structural defects in titan-germany alloys system is discussed. Defects are created by charged particles radiation. Positrons are sensitive to radiation defects because of influencing on electronic structure of metals. Knowing behavior of positrons and annihilation parameters changing, it is possible to trace and predict evolution of structural violations. Such information is extremely important both for fundamental problems of education and accumulation of radiation defects, and for development of recommendations for their practical use.

Key words: metals, alloys, defects, an alpha-particles, positron, electron, annihilation.

Language: Russian

Citation: Mukashev KM, Yar-Mukhamedova GS, Shadinova KS, Shoinbaeva GT (2015) ANNIHILATION OF POSITRONS IN RADIATION DAMAGED TITAN ALLOYS. ISJ Theoretical \& Applied Science 05 (25): 38-43.

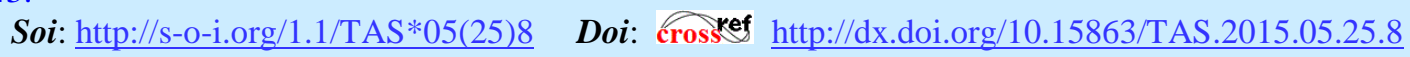

\section{АННИГИЛЯЦИЯ ПОЗИТРОНОВ В РАДИАЦИОННО ПОВРЕЖДЕННЫХ СПЛАВАХ ТИТАНА}

Аннотация: Обсуждается возможность применения метода позитронной спектроскопии для исследования структурных дефектов в системе сплавов титан-германий. Дефекты создаются путем облучения заряженными частицами. Позитроны оказываются чувствительными к радиационным дефектам вследствие влияния последних на электронную структуру металлов. Зная поведение позитронов $u$ по изменению аннигиляциионных параметров, можно отслеживать и прогнозировать эволюиию структурных нарушений. Такая информачия крайне важна как с точки зрения фундаментальных проблем образования и накопления радиационных дефектов, так и с позиции выработки рекомендаций для их практического использования.

Ключевые слова: металлы, сплавы, дефекты, альфа - частицы, позитрон, электрон, аннигиляциия.

\section{Введение}

В физике радиационных повреждений правильная постановка задачи и грамотный выбор объектов исследований имеют решающее значение в достижении поставленной цели, особенно если основным средством исследований является метод позитронной спектроскопии. По данным [1] по степени изученности с точки зрения проявления эффекта захвата позитронов, все металлы можно было разделить на три класса. Первый класс был составлен из 18 элементовметаллов, проявляющих эффект захвата. Следующая, более малочисленная группа из 14 элементов, в том числе (Ті и $\mathrm{Ge}$ ), считается не проявляющими эффект захвата позитронов. Правда, из каких соображений авторы работы [1] относят те или иные металлы к классу проявляющих или не проявляющих эффект захвата позитронов, остается не выясненными.

На основании всестороннего анализа этих данных, был выработан следующий критерий отбора объектов исследований методами электронно-позитронной аннигиляции (ЭПА). Поскольку тщательный, критический анализ на присутствие эффекта захвата позитронов для всех перечисленных металлов на представляется возможным, было решено отобрать среди элементов 2-класса такой базовый металл, 
который во всех отношениях был доступным и перспективным конструкционным материалом, без особой сложности и затрат образовывал систему бинарных сплавов с представителями как среди своего, так и других классов. Именно всем этим требованиям во всех отношениях удовлетворяет титан, обладающий многими уникальными свойствами, которых невозможно найти в других металлах. В качестве второго компонента при изготовлении бинарных сплавов был выбран Ge из числа элементов 2 - класса. Для исследования характера радиационной повреждаемости при одновременном установлении эффекта захвата позитронов были приготовлены сплавы на основе иодидного титана, содержащие $0 ; 1,2 ; 2,5 ; 3,3 ;$ и 4,1 ат. \% Ge. Концентрации второго компонента были выбраны так, чтобы все сплавы находились в области твердых растворов. По форме образцы имели вид диска диаметром 15 мм, толщиной 1 мм. Исходное состояние материалов достигалось отжигом при температуре $900^{\circ} \mathrm{C}$ и вакууме $10^{-7}$ торр. в течение 1 часа.

\section{Методика эксперимента}

Поскольку методы позитронной аннигиляции чувствительны в основном к дефектам вакансионного характера, то здесь ставилась задача сохранения в ходе облучения исследуемого материала дефектов именно этого типа. Вакансионные дефекты становятся подвижными при температуре выше $100^{\circ} \mathrm{C}$ [2]. Следовательно, при облучении необходимо обеспечить температуру образца не выше указанной, что в принципе является не сложной задачей. Междоузельные атомы в этих условиях мигрируют к стокам и на результаты исследования методами ЭПА не оказывают существенного влияния [3].

Обычно при облучении металлов заряженными частицами, последние теряют свою энергию на образование радиационных дефектов и на нагрев образца. Температура образцов измерялась привариванием термопары группы хромель-алюмель к защемленным краям, т.е. при облучении термопара находилась вне поля действия ионизирующего излучения. Облучение образцов ионами гелия на изохронном ускорителе У-150 проводилось в воздушной атмосфере с водоохлаждаемым основанием с принудительным воздушным обдувом образца или парами жидкого азота. При интенсивности альфа-частиц $(1,5-2) \cdot 10^{12} \mathrm{~cm}^{-2} \mathrm{c}^{-1}$ и энергии $\mathrm{E}=50$ МэВ, температура образца не превышала 60$70^{\circ} \mathrm{C}$. Измерив среднее значение тока пучка $I$, можно определить флюенс частиц в течение заданного времени $t$ или время, необходимое для достижения требуемой дозы при данной величине тока пучка через образец:

$$
D=6,25 \cdot 10^{12} \text { It } / S,
$$

где $S$ - площадь образца.

\section{Обсуждение результатов}

Для интерпретации результатов исследований были использованы следующие аннигиляционные параметры: Fперераспределение вероятности аннигиляции позитронов между электронами проводимости и связанными электронами, извлекаемая в результате обработки спектра угловой корреляции аннигиляционного излучения и угол, соответствующий импульсу Ферми $\theta_{\mathrm{F}}[2,3]$. Кинетика накопления радиационных дефектов в результате облучения альфа-частицами выбранной системы сплавов $\mathrm{Ti}-\mathrm{Ge}$ изучалась при флюенсах $10^{14} ; 3,2 \cdot 10^{14} ; 3,2 \cdot 10^{15}$ и $10^{16} \mathrm{~cm}^{-2}$. При этом дозовая зависимость радиационной повреждаемости системы изучалась при фиксированной энергии частиц $\mathrm{E}=29$ МэВ и интенсивности пучка $1,5 \cdot 10^{12} \mathrm{~cm}^{-2} \mathrm{c}^{-1}$. Наряду с этим, эти же исследования повторились при энергии частиц $\mathrm{E}=50$ МэВ при флюенсе $5 \cdot 10^{15}$ $\mathrm{cm}^{-2}$. Температура образцов во время облучения поддерживалась в пределах 60 - $70^{\circ} \mathrm{C}$. После облучения при очередном флюенсе частиц, проводилось измерение спектра угловой корреляции с последующей его обработкой с целью выделения структурно - чувствительных параметров, как $F$ и $\theta_{\text {F, }}$ а также соответствующие им относительные изменения $\Delta F$ и $\Delta \theta_{\text {F. }}$. Результаты выполненных исследований сведены в таблицу 1. Не трудно заметить, что с ростом флюенса в поведении аннигиляционных параметров наблюдаются заметные изменения уже при самом низком его уровне - $10^{14} \mathrm{~cm}^{-2}$. Неуклонный рост вероятности аннигиляции позитронов, следовательно, и эффективности захвата позитронов с флюенсом имеет место практически для всех изученных сплавов.

Наибольшее значение эффективности захвата позитронов наблюдается при облучении $\alpha$-частицами с $E=50$ МэВ, результаты которых приведены здесь же для сравнения. При этом флюенс частиц был на порядок меньше, чем при облучении $\alpha$-частицами с $E=29$ МэВ. Сравнительно равномерное снижение угла Ферми $\theta_{\mathrm{F}}$ с ростом флюенса облучения можно заметить для первых двух составов, в то время как для сплава $\mathrm{Ti}-3,1 \quad$ ат.\% $\mathrm{Ge}$ после обычного уменьшения при первых двух значениях флюенса $10^{14}$ и $3,2 \cdot 10^{14} \mathrm{~cm}^{-2}$ наблюдается постепенное его повышение, который достигает значения $\theta_{\mathrm{F}}=5,87$ мрад. при флюенсе $10^{16} \mathrm{~cm}^{-2}$. Однако оно все же остается ниже значения для отоженного состояния $\theta_{\mathrm{F}}=6,0$ мрад. Безусловно, первоначальное уменьшение $\theta_{\text {F }}$ в этом случае, очевидно, связано с образованием дефектов, обладающих более низкой электронной 
плотностью. Но последующий рост $\theta_{\mathrm{F}}$ с увеличением флюенса может быть вызван с радиационным отжигом, тогда в этом случае

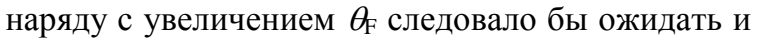
снижение параметра $F$, чего не наблюдается на практике. Поэтому, вероятно, следует допустить, что образованию радиационных повреждений в этом сплаве сопутствует инициирование частиц выделений вследствие распада пересыщенного твердого раствора. Согласно данным работ [4,5] при распаде сплава Тi-3,1 ат.\% Ge образуется фаза $\mathrm{Ti}_{5} \mathrm{Ge}_{3}$. Электронная плотность таких выделений должна отличаться от таковой для $\alpha-и \quad \beta-$ Ti. Следовательно, одновременное

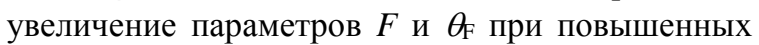
дозах может указывать на захват позитронов частицами фазы выделений.

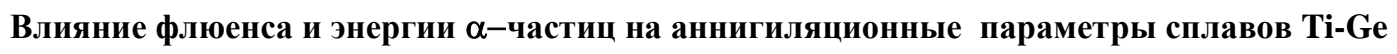

\begin{tabular}{|c|c|c|c|c|c|c|}
\hline $\begin{array}{c}\text { Состав, } \\
\text { ат. } \%\end{array}$ & $\begin{array}{c}\text { Энергия } \\
\text { МэВ }\end{array}$ & $\begin{array}{c}\text { Флюенс, } \\
\mathrm{cm}^{-2}\end{array}$ & $F$ & $\begin{array}{l}\Delta F \\
\%\end{array}$ & $\begin{array}{c}\theta_{F}, \\
\text { мрад. }\end{array}$ & $\begin{array}{l}\Delta \theta_{\mathrm{F}} \\
\%\end{array}$ \\
\hline \multirow{7}{*}{$\mathrm{Ti}-0,8 \mathrm{Ge}$} & - & отож. & 0.26 & - & 6.40 & - \\
\hline & 29 & $10^{14}$ & 0.32 & 23 & 5.82 & 6.2 \\
\hline & 29 & $5 \cdot 10^{14}$ & 0.48 & 85 & 5.68 & 11.2 \\
\hline & 29 & $10^{15}$ & 0.50 & 92 & 5.80 & 9.4 \\
\hline & 29 & $5 \cdot 10^{15}$ & 0.55 & 112 & 5.57 & 10.9 \\
\hline & 29 & $10^{16}$ & 0.71 & 173 & 5.39 & 15.8 \\
\hline & 50 & $5 \cdot 10^{15}$ & 0.78 & 200 & 5.57 & 10.9 \\
\hline \multirow{7}{*}{$\mathrm{Ti}-1,5 \mathrm{Ge}$} & - & отож. & 0.27 & - & 6.33 & - \\
\hline & 29 & $10^{14}$ & 0.35 & 30 & 6.00 & 5.2 \\
\hline & 29 & $5 \cdot 10^{14}$ & 0.40 & 48.1 & 5.83 & 7.9 \\
\hline & 29 & $10^{15}$ & 0.51 & 89 & 5.48 & 13.4 \\
\hline & 29 & $5 \cdot 10^{15}$ & 0.56 & 107 & 5.57 & 12.0 \\
\hline & 29 & $10^{16}$ & 0.67 & 148 & 5.39 & 14.8 \\
\hline & 50 & $5 \cdot 10^{15}$ & 0.76 & 181 & 5.57 & 12.0 \\
\hline \multirow{7}{*}{$\mathrm{Ti}-3,1 \mathrm{Ge}$} & - & отож. & 0.41 & - & 6.00 & - \\
\hline & 29 & $10^{14}$ & 0.47 & 145 & 5.76 & 4.0 \\
\hline & 29 & $5 \cdot 10^{14}$ & 0.56 & 37 & 5.57 & 7.2 \\
\hline & 29 & $10^{15}$ & 0.52 & 27 & 5.66 & 5.7 \\
\hline & 29 & $5 \cdot 10^{15}$ & 0.60 & 46 & 5.83 & 2.8 \\
\hline & 29 & $10^{16}$ & 0.77 & 88 & 5.87 & 2.2 \\
\hline & 50 & $5 \cdot 10^{15}$ & 0.84 & 105 & 5.57 & 7.2 \\
\hline \multicolumn{2}{|c|}{ Погрешности \pm} & - & 0.01 & 0.5 & 0.05 & 0.1 \\
\hline
\end{tabular}

Анализ результатов облучения сплавов Ті, содержащих 0,8 и 1,5 ат.\% $\mathrm{Ge}$, указывает на полную идентичность характера изменений параметров $F$ и $\theta_{\text {F }}$ для них, для которых отсутствует стремление к насыщению при увеличении флюенсов $\alpha$-частиц до $10^{16} \mathrm{~cm}^{-2}$. Если допустить, что увеличение флюенса $\alpha$-частиц влечет за собой только повышение концентрации центров захвата позитронов в этих сплавах, то это не должно отражаться на значении $\theta_{\text {F. }}$ Одновременное уменьшение угла Ферми $\theta_{\mathrm{F}}$ с повышением флюенса при постоянной тенденции к росту вероятности аннигиляции $F$ можно интерпретировать только соответствующим увеличением эффективности захвата ловушками позитронов. Вероятно, такими ловушками могут быть в данном случае образование связанных состояний вакансияпримесь, эффективность к захвату которых должна быть выше простых вакансионных дефектов и их скоплений. Общее приращение аннигиляционного параметра $\mathrm{F}$ при увеличении флюенса от $10^{14}$ до $10^{16} \mathrm{~cm}^{-2}$ составило для сплава, содержащего 0,8 ат.\% Ge, $\Delta F=173 \%$. Для второго сплава $\mathrm{Ti}-1,8$ ат.\% Ge оно оказалось равным уже $148 \%$ и в последнем случае - около $88 \%$. Такая же тенденция наблюдается в отношении изменения угла Ферми (см. таблицу 1). И самое главное, данная закономерность сохраняется при облучении сплавов $\alpha$-частицами с $E=50$ МэВ, но приращение вероятностей $\Delta F$ соответственно в каждом случае уже составило $200 ; 181$ и 105\% при постоянном значении флюенса $5 \cdot 10^{15} \mathrm{~cm}^{-2}$. Разумеется, для достижения таких значений аннигиляционного параметра следовало бы довести флюенс 29 МэВ-ных $\alpha$-частиц еще на один порядок от достигнутого уровня $10^{16} \mathrm{~cm}^{-2}$. Следовательно, при постоянном 
значении $\theta_{\mathrm{F}}=5,57$ мрад. для всех трех сплавов, полученном при облучении $\alpha$-частицами более высокой энергии, значительное (почти в 1,8 раза) возрастание $\Delta F$ является причиной соответствующего повышения концентрации радиационных дефектов. Данное обстоятельство является основанием полагать, что радиационные дефекты в исследуемых материалах имеют сходную конфигурацию в данном энергетическом диапазоне. Но значительное возрастание эффективности захвата позитронов по параметру $F$ с изменением энергии бомбардирующих частиц указывает на роль ядерных реакций в процессах радиационного дефектообразования материалов [6], тем более, что радиационная активность последних по данным результатов дозиметрического контроля при облучении $\alpha$-частицами с $E=50$ МэВ значительно выше, чем после облучения частицами с $E=29$ МэВ. Таким образом, достигнутые значение аннигиляционных параметров, полученных при облучении $\alpha$-частицами сплавов $\mathrm{Ti}$, являются не предельными и находятся еще достаточно далеко от насыщения.

Кинетику накопления радиационных дефектов в сплавах Ti-Ge в зависимости от флюенса $\alpha$-частиц можно оценить по изменению эффективности захвата позитронов, определяемой по коэффициентом захвата:

$$
K=\left(F_{\alpha}-F_{0}\right) /\left(F_{\mathrm{m}}-F_{\alpha}\right)
$$

где $F_{\alpha}-$ текущее значение параметра; $F_{0}-$ значение параметра для отожженного состояния материала; $F_{\mathrm{m}}-$ максимальное значение параметра в изучаемом интервале.

Характер изменения данного коэффициента в зависимости от флюенса $\alpha$-частиц для Ті (1) и Ti-3,1 ат.\% Ge представлен на рис. 1. Видно, что коэффициент захвата позитронов возрастает для рассматриваемых материалов примерно с одинаковым темпом, а характер изменения практически не зависит от концентрации легирующего элемента.

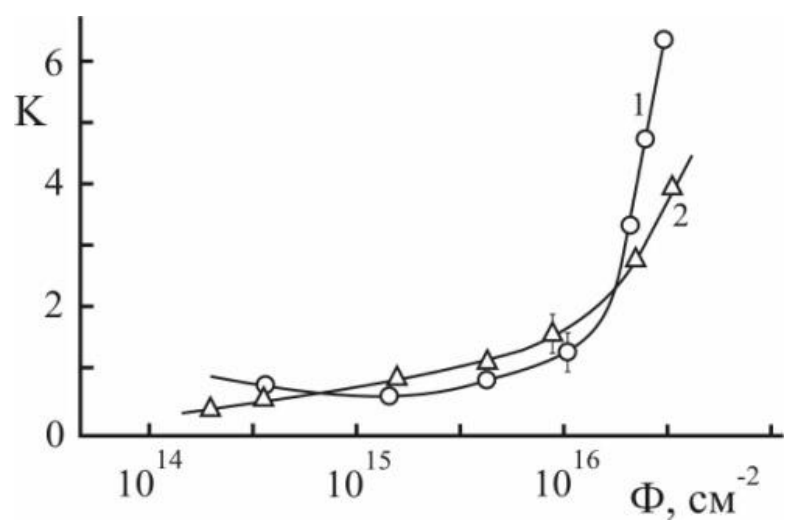

Рисунок 1 - Дозовая зависимость эффективности захвата позитронов для сплавов Тi (1) и Ti 3,1 ат.\% Ge (2).

В то же время скорость роста коэффициента $K$ для сплава несколько отстает от таковой для $\mathrm{Ti}$, причиной которого может быть изменение конфигурации радиационных дефектов, приводящее к соответствующему перераспределению электронной плотности в поврежденных областях.

В интервале флюенсов $10^{14} \div 5 \cdot 10^{15} \mathrm{~cm}^{-2}$ скорость роста коэффициента захвата позитронов существенно ниже, чем при дозах, превышающих $5 \cdot 10^{15} \mathrm{~cm}^{-2}$. Данный участок представляет собой инкубационный период накопления радиационных дефектов, выше которого процесс накопления дефектов растет стремительно. Но поскольку дефекты в данном случае носят вакансионный характер, то накопление в начале идет по принципу кластеризации, а в дальнейшем могут объединяться в микропоры. Характерным признаком микропор является ее критический радиус, которого они достигают флуктуационным способом за счет поглощения точечных дефектов, в результате чего они растут в размере интенсивно. Правда, не все поры одновременно достигают критического радиуса, и в этом случае начинается новая стадия процесса распухания - растворение мелких пор более крупными. Одновременно рождающиеся под облучением вакансии и междоузельные атомы (МУА) диффундируют к стокам. Если МУА захватываются порами, скорость роста последних резко замедляется, что влечет за собой изменение функции распределения пор по размером. В этом случае изменение объема металла в процессе распухания описывается выражением [7-10]:

$V(t)=A\left(D_{V} C_{V} R_{S}\right)^{1 / 3} \sqrt{G \cdot \rho_{d}\left(Z_{i}-Z_{V}\right)} \cdot t^{5 / 6}$ откуда видно, что процесс распухания зависит от температуры облучения $V \sim\left(D_{\mathrm{V}} C_{\mathrm{V}}\right) /^{1 / 3} ;$ скорости 
генерации дефектов $V \sim \sqrt{G}$ и времени облучения $V \sim t^{5 / 6}$. Таким образом, с течением времени облучения, т.е. от флюенса частиц, распухание растет без признаков насыщения, что и наблюдается в данном случае (рис. 2). Очевидно, этот процесс идет тем эффективнее, чем ниже энергия дефектов упаковки металла, а изучаемые сплавы титана относятся именно классу таких материалов. Данное обстоятельство хорошо проявляется в характере плотности распределения электронов, о чем пойдет речь позже.

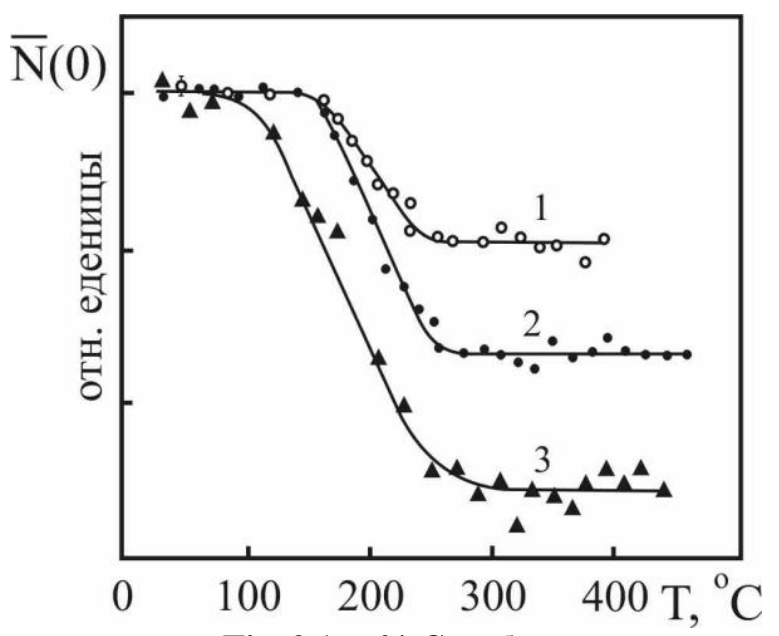

Рисунок 2 - Отжиг дефектов в сплавах Ti - 3.1ат.\% Ge, облученных $\alpha$ - частицами различной энергии и флюенса

1. $\mathrm{E}=\mathbf{2 9}$ МэВ, $\Phi=5 \cdot 10^{15} \mathrm{~cm}^{-2}$;

2. $\mathrm{E}=29 \mathrm{MэB}, \Phi=5 \cdot 10^{16} \mathrm{~cm}^{-2}$

3. $\mathrm{E}=50 \mathrm{MэB}, \Phi=\mathbf{5} \cdot 10^{15} \mathrm{~cm}^{-2}$

Сходность конфигурации образующихся радиационных дефектов в сплавах при облучении $\alpha$-частицами проявляется в результатах изохронного отжига, представленных на рис. 2. Видно, что независимо от флюенса и энергии последних, наблюдается только одна стадия возврата, свидетельствующая о присутствии в сплавах только одного типа структурных нарушений. Увеличение как флюенса (с $5 \cdot 10^{15}$ до $10^{16} \mathrm{~cm}^{-2}$ ), так и энергии частиц (с 29 до 50 МэВ) приводят лишь к повышению концентрации дефектов. Несколько затянутый характер завершения стадии отжига при облучении $\alpha$ частицами с $E=50$ МэВ по сравнению с $E=29$ МэВ является следствием наличия отличительных черт в характеристиках, прежде всего - конфигурациях радиационных повреждений, созданных за счет упругих взаимодействий без образования или незначительно отличающихся от вакансионных скоплений субкаскадов (при $E_{\alpha}=29$ МэВ, кривые $1,2)$ и за счет тех же упругих взаимодействий с образованием значительных субкаскадов и ядерных реакций $\left(E_{\alpha}=50\right.$ МэВ, кривая 3$)$.

В то же время найденные по выше изложенной методике значения энергии активации миграции радиационных дефектов в изученных сплавах в первом случае $E_{\mathrm{a} 1}=1.40-$
1.42 и $E_{\mathrm{a} 2}=1.50-1.55$ эВ - во втором, лишний раз может служить подтверждением указанных предположений. Кроме того, из анализа приведенных кривых отжига можно извлечь еще одну крайне важную информацию. Чем выше концентрация радиационных дефектов в сплаве, тем ниже начало температуры отжига, т.е. с возрастанием флюенса (от $5 \cdot 10^{15}$ до $10^{16} \mathrm{~cm}^{-2}$ ) при постоянной энергии $\alpha$-частиц (29 МэВ) или энергии (с 29 до 50 МэВ) при постоянном флюенсе $\left(5 \cdot 10^{15} \mathrm{~cm}^{-2}\right)$ начало возврата все более смещается в сторону низких температур. Вероятно, с увеличением концентрации точечных дефектов в кристалле снижаются те потенциальные барьеры, которые препятствуют активационным процессам, связанным с перемещением ионов. Одновременно с этим, очевидно, уменьшается и энергия связи между ними. Последнее в свою очередь ведет к снижению температурного порога миграции дефектов, что и отражается на кривых изохронного отжига. Полный отжиг структурных нарушений в сплавах, облученных $\alpha$ - частицами с $E=29$ МэВ завершается в области температуры $300^{\circ} \mathrm{C}$. 
Impact Factor ISRA (India) $\quad=\mathbf{1 . 3 4 4}$

Impact Factor ISI (Dubai, UAE) $=\mathbf{0 . 8 2 9}$

based on International Citation Report (ICR)

Impact Factor GIF (Australia) $\quad \mathbf{0} \mathbf{0 . 3 5 6}$ $\begin{array}{lr}\text { Impact Factor JIF } & =\mathbf{1 . 5 0 0} \\ \text { Impact Factor SIS (USA) } & =\mathbf{0 . 9 1 2} \\ \text { Impact Factor PИНЦ }(\text { Russia }) & =\mathbf{0 . 1 7 9} \\ \text { Impact Factor ESJI }(\text { KZ) } & =\mathbf{1 . 0 4 2}\end{array}$

из анализа полученных данных, можно сделать предположение, что облучение, как и деформация, может инициировать такие структурные нарушения, потенциал захвата которых для позитронов гораздо больше, чем для простых дефектов типа вакансии и дислокации. Но радиационные явления, сопровождающие эти превращения, очевидно, представляют для термолизованных позитронов более глубокие потенциальные ямы захвата, чем те, которые сопутствуют полиморфным превращениям, инициированным деформацией.

\section{References:}

1. Tanigawa S, Hinode K, Owada N, Doyama M (1979) The phenomenological Interpretation of positron lifetime and Trapping effect in metals with the special reference to the state of Hydrogen. Proc. 5th. Int. Conf. of Positron Annihilation. Japan, Tokyo.159.

2. Mukashev KM, Tronin BA (2010) Defekty vodorodnogo proiskhozhdeniya $\mathrm{V}$ splavakh tugoplavkikh metallov i annigilyatsiya pozitronov. Izvestiya VUZov. Seriya fizika, Tomsk. 55 .

3. Mukashev KM (2009) Fizika medlennykh pozitronov i pozitronnaya spektroskopiya. Almaty. 507.

4. Novoselova AV (1987) Fazovye diagrammy, ikh postroenie i metody issledovaniya. Moscow, MGU. 151.

5. Quillan M (1956) Titanium.- Bettelworth. 235.

6. Gindin IA, Lapiashvili ES, Leselidze IK (1978) Vliyanie radiatsionnykh defektov na protsessy programmnogo uprochneniya titana //
Reaktornoe materialovedenie. Moscow. TsNIIAtominform.T. 2. 317.

7. Ibragimov SS, Reutov VF, Vagin SP, Botvin KV (1987) Radiatsionnye povrezhdeniya v Mo, obluchennom protonami i $\alpha$ - chastitsami. Fizika i.KhOM., No1. 3.

8. Dekhtyar IY, Levina DA, Mikhalenkov VS (1994) Annigilya ciyapozitronov s elektronami v plasticheski dephormirovannikh metallakh. Doklady Akademii Nauk Ukrainy. No4.

9. Faraci G, Pennisi A (2002) The annihilation spectrum is temperature independent. Lett. Nuovo Cim. No1.

10. Mukashev KM, Yar-Mukhamedova GS, Shadinova KS (2015) RADIATION AND ANNEALING PROCESSES IN BINARY ALLOYS OF TITANIUM. ISJ Theoretical \& Applied Science 04 (24): 10-14. Soi: http://s-oi.org/1.1/TAS*04(24)2 Doi: http://dx.doi.org/10.15863/TAS.2015.04.24.2 